\title{
The Probationary Period as a Screening Device: The Monopolistic Insurer
}

\author{
JAAP SPREEUW \\ Cass Business School, Faculty of Actuarial Science and Statistics, 106 Bunhill Row, London EC1Y 8TZ, UK
}

Received May 17, 2003; Revised February 24, 2004

\begin{abstract}
Stiglitz [1977], considering asymmetry of information in a monopolistic insurance market and the monetary deductible as a screening device, shows that an equilibrium is always of a separating type. High risks buy complete insurance whilst low risks buy partial insurance. In this paper, we show that a pooling equilibrium may exist if a probationary period, rather than the partial coverage in monetary terms, is used as a screening device.
\end{abstract}

Key words: probationary periods, adverse selection, monopoly, separating equilibrium, pooling equilibrium

JEL Classification No.: D42, D82

\section{Introduction}

In insurance markets with adverse selection and two different risk types, insurers can implement a screening tool to separate low risk and high risk individuals. The most common device is the monetary deductible. Stiglitz [1977] has shown that, if a monopolistic insurer applies this instrument, an equilibrium is always of a separating type. High risks buy full coverage and low risks buy partial coverage, or no insurance at all. In other words, a pooling equilibrium cannot exist.

An alternative screening device, which will be the theme of this paper, is the probationary period. Such a device excludes coverage for events that occur during a predefined period after the inception of the policy. The method, aiming to rule out preexisting conditions, has found applications in some dental and medical policies. Besides, over recent years, it has gained popularity among Dutch group life companies as a consequence of new legislation concerning the medical examination of employees. By the new law which came into force at the beginning of 1998, insurance companies are strongly restricted in their possibilities to perform medical tests on individual members of a group life scheme. Therefore, they cannot determine whether any member has a serious disease. A probationary period may then be an appropriate instrument to keep out individuals who are likely to make a claim soon after inception.

Several characteristics of the probationary period, its impact on the expected utility of individuals among others, have been investigated in Eeckhoudt et al. [1988]. They prove that, in a fully competitive market, the equilibrium under symmetric information involves full coverage for any risk class, similarly to the monetary deductible case, provided that the 
probability of incurring an accident is smaller than one. The authors' main conclusion is that most of the basic properties of the above mentioned monetary deductibles do not carry over to probationary periods.

Fluet [1992] applies the concept of a probationary period in a competitive insurance market with asymmetric information. He adopts the screening device of a time-dependent monetary deductible. Assuming that the proportion of high risk agents is large enough, the high risks buy full coverage, while the low risks buy partial coverage in monetary terms.

This paper deals with a monopolistic insurance market and focuses on the probationary period, rather than the monetary deductible, as an instrument to let individuals self-select. In this respect, the approach is less general than Fluet [1992] as contracts incorporating both time and monetary deductibles are not considered. It should be stated, however, that a monetary deductible always implies some non-linear pricing which may be difficult to implement in practice. Moreover, Fluet [1992] has shown that the combination of both devices makes it difficult to draw conclusions, unless restricting assumptions are being made. By concentrating on the probationary period, one can get an idea of possible equilibria resulting if, just as in Fluet [1992], allowance is made for partial coverage in monetary terms.

We will show that, contrary to the analysis in Stiglitz [1977], a pooling equilibrium, with complete coverage for both risk types, may exist. To demonstrate this, we will follow an approach which will appeal to the reader's intuition by comparing the monetary deductible with the probationary period.

The model will be described in Section 2, where also the basic assumptions will be listed and the details about the main example in this paper will be given. Section 3 deals with the two risk types separately. We will show that, for full coverage, the absolute value of the slope of any individual's indifference curve exceeds the firm's corresponding marginal profit. This is an important property which will be used throughout the remainder of this contribution. The next step, involving adverse selection, will be taken in Section 4. We will show that, if the insurer's strategy is restricted to pooling contracts, full coverage may be optimal. This is a result which contradicts Stiglitz' findings. In Section 5, the restriction of offering pooling contracts only is dropped and hence the insurer can offer any pair of policies. It is shown that even then a pooling contract may be optimal. Under the assumptions stated in this paper, such a pooling contract would involve complete coverage. Conclusions and recommendations for further research are given in Section 6.

\section{The basic assumptions and the nature of a probationary period}

In this section we will start with an overview of the general assumptions and definitions in Section 2.1. Thereafter, in Section 2.2, we will indicate the relationship between the time-of-accident of the high risks and low risks and motivate our choice.

\subsection{General assumptions}

The basic assumptions are mainly derived from Fluet [1992]. For the ease of exposition they are listed below: 
- A population consists of two classes of risk, namely the high risks and the low risks. In the remainder of this paper all variables pertaining to high risk and low risk individuals will be accompanied by the subscripts $H$ and $L$, respectively. All individuals have an initial, non-random, wealth of $W$.

- All individuals within the population are identical, except with respect to the probability of having an accident in the period $[0, n]$, where 0 is the current time and $n$ is real valued and positive. By "accident" we mean the occurrence of a certain unfavorable event, which can e.g. be injury or death. In case an individual is faced with an accident, there is a welfare loss $D$. The probability of having an accident for an individual of risk class $i$ is denoted by $\eta_{i}, i \in\{H, L\}$, with $\eta_{L}<\eta_{H}<1$. It is assumed that an accident can occur to each individual at most once.

- All risks are insurable.

- The population consists of $N$ individuals, of which $N_{H}$ and $N_{L}$ belong to the category of high risks and low risks respectively. Hence $N=N_{H}+N_{L}$. The proportion of high risks among the entire population is denoted by $\rho$, so $N_{H}=\rho N$.

- The time at which any accident occurs is perfectly observable by both the individual concerned and the company.

- The probability for an individual of risk class $i, i \in\{H, L\}$, of facing an accident before time $t(0 \leq t \leq n)$ is denoted by $F_{i}(t)$ (hence $\left.F_{i}(n)=\eta_{i}\right)$, and it is assumed to be differentiable in $[0, n]$, with derivative $f_{i}(t)$. All individuals fully know these probabilities. These probabilities are exogenous, so that the risk of moral hazard is non-existent.

- To each individual, the same utility function $U(\cdot)$ applies, which is assumed to be increasing, strictly concave, twice continuously differentiable and independent of time.

- Only expected wealth at time $n$ matters and all interest rates are set equal to zero. Any individual's concern is to maximize expected utility.

- Insurance companies are risk neutral (implying that each company's utility function is linear). Their concern is to maximize expected profit.

- Insurance companies have enough resources to offer any set of contracts, which result in a nonnegative expected profit.

- There are no transaction costs involved in the supply of insurance and no administrative costs for the insurance business. Nor are there costs of obtaining classification information on a potential insured when it is possible to do so.

- Contracts are specified by $(t, P)$, with $t$ and $P$ denoting the probationary period and the premium respectively. For the given contract, no indemnity is paid if an accident occurs in the period $[0, t]$, nor will the premium $P$, to be paid at time 0 , be refunded to the insured. On the other hand, if an accident occurs during the period $(t, n]$, the insured will get a benefit equal to $D$.

- We denote the expected utility resulting from taking out the policy $(t, P)$ by $E_{i}(t, P)$. This implies:

$$
E_{i}(t, P)=F_{i}(t) U(W-P-D)+\left(1-F_{i}(t)\right) U(W-P) ; \quad i \in\{1,2\} .
$$

The special case case of no insurance is denoted by $E_{i}$, so:

$$
E_{i}=E_{i}(n, 0)=\eta_{i} U(W-D)+\left(1-\eta_{i}\right) U(W), \quad i \in\{H, L\} .
$$




\subsection{Relationship between distribution functions of time-of-loss}

We have already defined $F_{H}(t)$ and $F_{L}(t)$, the c.d.f.'s of time of loss, in the previous subsection. We will now establish a relationship between those functions by assuming that the ratio between $F_{H}(t)$ and $F_{L}(t)$ is fixed, i.e.:

$$
F_{H}(t)=b F_{L}(t), \quad \text { with } b>1 ; 0 \leq t \leq n .
$$

This strict inequality between $F_{H}(t)$ and $F_{L}(t)$ ensures that all contracts which will be purchased by the low risks are also acceptable for the high risks. Consider the contract $(t, P)$. Then we have:

$$
\begin{aligned}
E_{H}(t, P)-E_{H}= & U(W-P)-U(W)+b\left(\eta_{L}(U(W)-U(W-D))\right. \\
& \left.-F_{L}(t)(U(W-P)-U(W-P-D))\right) \\
> & U(W-P)-U(W)+\eta_{L}(U(W)-U(W-D)) \\
& -F_{L}(t)(U(W-P)-U(W-P-D)) \\
= & E_{L}(t, P)-E_{L} \\
\geq & 0 .
\end{aligned}
$$

We will see later in this paper that the optimal strategy for the monopolistic insurer will then have some properties in common with the one derived in Stiglitz [1977].

A fixed ratio between $F_{H}(t)$ and $F_{L}(t)$ is not necessary to achieve the inequality (4). We could replace the factor $b$ in (3) by a function depending on $t$, say $b(t)$ with $b(t) \geq 1$. However, we have chosen for a fixed factor as it will make the computations relatively straightforward.

\section{Indifference curves and iso-profit curves}

In this section, we will show that, at any point of full coverage an individual's marginal rate of substitution of premium for time deductible, exceeds the firm's marginal profit. We will follow an intuitive approach. Recall that, in case of a monetary deductible these two quantities are always equal to each other.

We define $\Gamma(t, P)$ as the expected profit resulting from offering a contract $(t, P)$ to an individual. This gives

$$
\Gamma(t, P)=P-(\eta-F(t)) D .
$$

The marginal profit, in terms of the probationary period, is equal to

$$
\frac{\partial \Gamma(t, P)}{d t}=f(t) D
$$

The individual's marginal rate of substitution of premium for probationary period (the slope 
of the indifference curve) is equal to

$$
\begin{aligned}
-\frac{d P}{d t} & =\frac{\frac{\partial E_{i}(t, P)}{d t}}{\frac{\partial E_{i}(t, P)}{d P}} \\
& =f(t) D \frac{\frac{U(W-P)-U(W-P-D)}{D}}{F(t) U^{\prime}(W-P-D)+(1-F(t)) U^{\prime}(W-P)} \\
& =f(t) D \frac{U^{\prime}(W-P-\beta)}{F(t) U^{\prime}(W-P-D)+(1-F(t)) U^{\prime}(W-P)},
\end{aligned}
$$

for some $\beta \in[0, D]$, in accordance with the mean value theorem.

For $t=0$ (full coverage), the above expression reduces to

$$
-\left(\frac{d P}{d t}\right)_{(t=0)}=f(0) D \frac{U^{\prime}(W-P-\beta)}{U^{\prime}(W-P)}>f(0) D .
$$

This inequality suggests that the effectiveness of the probationary period as a screening device can be quite poor. We will use this result in the next sections.

In Appendix A, it will be shown that full coverage is optimal in case of symmetric information (in Eeckhoudt et al. [1988] this has been shown for a competitive market).

\section{Optimal pooling contract}

Adopting a two steps approach, Stiglitz [1977] proves that an optimal strategy for the monopolistic firm always involves issuing two separating policies. Firstly, he considers a pooling contract with incomplete coverage, and concludes that such a strategy would always be inferior to offering a pair of different contracts, with the original pooling contract designed for the low risks and full coverage for the high risks.

Secondly, he establishes that a pooling contract with full coverage (the remaining option) can never be optimal, as it will always be inferior to at least some pooling contracts with incomplete coverage.

In this section, we will concentrate on the latter conclusion. Our aim is to show that, with the probationary period as screening device, offering full coverage could be optimal if the firm's choice were restricted to selling pooling contracts.

Assume that the insurer can only offer a pooling contract $(t, P)$. Let's denote the objective function by $\hat{\Gamma}(t, P)$, which is defined as the expected profit resulting from offering those contracts, divided by the total number of individuals, $N$. Then we have

$$
\begin{aligned}
\hat{\Gamma}(t, P) & =\frac{N P-N_{H}\left(\eta_{H}-F_{H}(t)\right) D-N_{L}\left(\eta_{L}-F_{L}(t)\right) D}{N} \\
& =P-(\rho b+(1-\rho))\left(\eta_{L}-F_{L}(t)\right) D .
\end{aligned}
$$

To maximize the profit, these contracts have to satisfy the binding reservation constraint for the low risks:

$$
E_{L}=E_{L}(t, P) .
$$


Notation 1: We denote the maximal premium the low risks and high risks are prepared to pay for full coverage by $P_{0}^{L}$ and $P_{0}^{H}$, respectively.

So we can express $P$ as a function of $t$. We will use the notation $P(t)$. This leads to $\hat{\Gamma}(t)$, denoting $\hat{\Gamma}(t, P)$ in (9) expressed as a function of $t$ only:

$$
\hat{\Gamma}(t)=P(t)-(\rho b+(1-\rho))\left(\eta_{L}-F_{L}(t)\right) D .
$$

We will analyze the function (11) by taking its derivative with respect to $t$. This returns:

$$
\begin{aligned}
d \frac{\hat{\Gamma}(t)}{d t}= & \frac{d P(t)}{d t}+(\rho b+1-\rho) f_{L}(t) D, \\
= & -f_{L}(t) D \cdot\left(\frac{\frac{U(W-P(t))-U(W-P(t)-D)}{D}}{F_{L}(t) U^{\prime}(W-P(t)-D)+\left(1-F_{L}(t)\right) U^{\prime}(W-P(t))}\right. \\
& -(\rho b+1-\rho)) .
\end{aligned}
$$

In accordance with the mean value theorem:

$$
\frac{U(W-P(t))-U(W-P(t)-D)}{D}=U^{\prime}(W-P(t)-\beta(t)),
$$

for some $\beta(t) \in[0, D], t \in[0, n]$. Note that, compared to the previous section, $P$ is a function of $t$, so $\beta$ is a function of $t$ as well. Consequently:

$$
U^{\prime}(W-P(t)-D) \leq U^{\prime}(W-P(t)-\beta(t)) \leq U^{\prime}(W-P(t))
$$

For $t=0$, expression (12) reduces to

$$
\left(d \frac{\hat{\Gamma}(t)}{d t}\right)_{(t=0)}=-f_{L}(0) D\left(\frac{U^{\prime}\left(W-P_{0}^{L}-\beta(0)\right)}{U^{\prime}\left(W-P_{0}^{L}\right)}-(\rho b+1-\rho)\right)
$$

which is negative for $\rho=0$ or $b=1$. By continuity, it is therefore negative at $t=0$ for $\rho$ and $b$ in a neighborhood of 0 and 1, respectively. This implies that, for such values of $\rho$ or $b$, the pooling contract with complete coverage is at least not the worst among all the pooling contracts. Recall that, for the monetary deductible as a screening device, the above expression is always positive, so complete coverage can never be optimal.

Furthermore, note that if $\eta_{L}$ is small, then $F_{L}(t)$ is small for each $t \in[0, n]$ and hence, again by continuity, we have for each $t \in[0, n]$ :

$$
U^{\prime}(W-P(t)-\beta(t)) \geq F_{L}(t) U^{\prime}(W-P(t)-D)+\left(1-F_{L}(t)\right) U^{\prime}(W-P(t))
$$


which implies that $d \frac{\hat{\Gamma}(t)}{d t}$ is negative everywhere for $\rho$ and $b$ in a neighborhood of 0 and 1 , respectively. It follows that the best pooling strategy may indeed be to provide full coverage.

Of course, the insurer's choice is not restricted to pooling contracts; the general case where the insurer can offer any pair of policies, is dealt with in the next section.

\section{The equilibrium}

Obviously, the firm's opportunities for a higher profit are enhanced if, unlike the previous section, its choice is not restricted to pooling contracts.

Note that, just as in Stiglitz [1977],

- any contract acceptable for the low risk is also acceptable for the high risks (as follows from Eq. (3)), and

- complete coverage is optimal in case of symmetric information (as shown in Appendix A).

This implies that, again just as in Stiglitz [1977], any strategy offering a pooling contract with incomplete coverage to both risk types, is inferior to offering the original pooling contract to the low risks and full coverage to the high risks. Assume that $\left(t_{L}, P_{L}\right)$ were to be sold to the low risks, then the premium for the high risks, denoted by $P_{H}$, would satisfy the following binding self-selection constraint for the high risks:

$$
U\left(W-P_{H}\right)=b F_{L}\left(t_{L}\right) U\left(W-P_{L}-D\right)+\left(1-b F_{L}\left(t_{L}\right)\right) U\left(W-P_{L}\right) .
$$

The Lagrangian to be optimized has the following shape:

$$
\begin{aligned}
\mathcal{L}= & \rho\left(P_{H}-\eta_{H} D\right)+(1-\rho)\left(P_{L}-\left(\eta_{L}-F_{L}\left(t_{L}\right)\right) D\right) \\
& +\gamma_{H}\left\{E_{H}\left(0, P_{H}\right)-E_{H}\left(t_{L}, P_{L}\right)\right\}+\lambda_{L}\left\{E_{L}\left(t_{L}, P_{L}\right)-E_{L}\right\} .
\end{aligned}
$$

In (18), the Lagrange multiplier $\gamma_{H}$ applies to the self-selection constraint for the high risks, shown in (17). Since it is binding, we have that $\gamma_{H}>0$.

The parameter $\lambda_{L}$ corresponds to the reservation constraint for the low risks. One of the first order conditions to be satisfied is:

$$
\begin{aligned}
\frac{\partial \mathcal{L}}{\partial P_{L}} & =(1-\rho)-\left(\lambda_{L}-\gamma_{H}\right)\left[F_{L}\left(t_{L}\right) U^{\prime}\left(W-P_{L}-D\right)+\left(1-F_{L}\left(t_{L}\right)\right) U^{\prime}\left(W-P_{L}\right)\right] \\
& =0
\end{aligned}
$$

which can only be satisfied if $\lambda_{L}>0$. It follows that the reservation constraint for the low risks is binding, i.e.

$$
E_{L}\left(t_{L}, P_{L}\right)=E_{L}
$$

Hence, we can rewrite the Lagrangian in (18) as an expected profit function, written in terms of only one of the variables $t_{L}, P_{L}$ or $P_{H}$ just by substituting (17) and (20) into (18). In the 
same way as in the previous section, we will express it as a function of $t_{L}$ only. Likewise, $P_{L}$ and $P_{H}$ are functions of $t_{L}$, so we will use the notation $P_{L}\left(t_{L}\right)$ and $P_{H}\left(t_{L}\right)$, respectively. Denote the profit function by $\hat{\Gamma}\left(t_{L}\right)$. Then, substitution leads to:

$$
\hat{\Gamma}\left(t_{L}\right)=\rho\left(P_{H}\left(t_{L}\right)-\eta_{H} D\right)+(1-\rho)\left(P_{L}\left(t_{L}\right)-\left(\eta_{L}-F_{L}\left(t_{L}\right)\right) D\right) .
$$

By taking the derivative with respect to $t_{L}$, we get the following expression:

$$
\begin{aligned}
& d \frac{\hat{\Gamma}\left(t_{L}\right)}{d t_{L}}=\rho d \frac{P_{H}\left(t_{L}\right)}{d t_{L}}+(1-\rho)\left(d \frac{P_{L}\left(t_{L}\right)}{d t_{L}}+f_{L}\left(t_{L}\right) D\right) \\
& =f_{L}\left(t_{L}\right) D\left(\begin{array}{c}
\rho\left(\frac{h\left(t_{L}\right)}{U^{\prime}\left(W-P_{H}\left(t_{L}\right)\right)}\right)+ \\
(1-\rho)\left(1-\frac{U^{\prime}\left(W-P_{L}\left(t_{L}\right)-\beta\left(t_{L}\right)\right)}{F_{L}\left(t_{L}\right) U^{\prime}\left(W-P_{L}\left(t_{L}\right)-D\right)+\left(1-F_{L}\left(t_{L}\right)\right) U^{\prime}\left(W-P_{L}\left(t_{L}\right)\right)}\right)
\end{array}\right),
\end{aligned}
$$

with

$$
h\left(t_{L}\right)=\left(b-\frac{k\left(t_{L}, b\right)}{k\left(t_{L}, 1\right)}\right) U^{\prime}\left(W-P_{L}\left(t_{L}\right)-\beta\left(t_{L}\right)\right)
$$

where

$$
k\left(t_{L}, c\right)=c F_{L}\left(t_{L}\right) U^{\prime}\left(W-P_{L}\left(t_{L}\right)-D\right)+\left(1-c F_{L}\left(t_{L}\right)\right) U^{\prime}\left(W-P_{L}\left(t_{L}\right)\right) \quad c \geq 1 .
$$

The first term of (22) is positive but has an upper bound.

Using the same argument as in the previous section, we observe the following:

- The second term is negative for $t_{L}=0$. Hence, for sufficiently small $\rho$, the strategy of offering the pooling contract with complete coverage may be superior to at least some of the separating menus.

- For sufficiently small $\eta_{L}$, the second term is negative for each $t_{L} \in[0, n]$. Hence, for sufficiently small $\rho$, the pooling full coverage contract may yield maximal profit for the insurer.

\section{Conclusions, final comments and recommendations for further research}

In this contribution, it has been confirmed that, just as in Eeckhoudt et al. [1988], many properties applying to the monetary deductible do not hold for the probationary period. An individual may perceive a contract with a probationary period as very inferior compared to full coverage.

This may explain why, in contradiction to the conclusions in Stiglitz [1977], a pooling contract with complete coverage may be superior to any other pooling contract. Offering such a contract may even be more profitable than offering any set of separating policies. A strategy involving a pooling full coverage contract can be optimal if the low risk's 
probability of incurring an accident, the differences between the low risks' and the high risks' probabilities, as well as the proportion of high risks within the entire population are all relatively low.

The analyses in this paper are based on an insurance market with one monopolistic firm. We intend to study the case of a competitive market and work out whether an equilibrium may be of a pooling type. This may enable to be more specific about the characteristics of equilibria, allowing for time-dependent monetary deductibles such as in Fluet [1992]. It may be interesting to note that, in case $F_{H}(t)=b F_{L}(t)$ (as considered in this paper), Fluet establishes that the monetary deductible for the low risks is constant over time. This implies that the separating menu does not involve a probationary period.

\section{Appendix A. Full coverage optimal in case of symmetry of information}

Adopting the definitions stated in the previous section, the insurer's expected profit for an individual contract $(t, P)$, denoted by $\Gamma(t, P)$, is equal to

$$
\Gamma(t, P)=P-(\eta-F(t)) D
$$

Regarding the contract $(t, P)$, we have the following binding reservation restriction for an individual with accident probability $\eta$ :

$$
F(t) U(W-P-D)+(1-F(t)) U(W-P)=\eta U(W-D)+(1-\eta) U(W) .
$$

In the remainder of this appendix, the right hand side of Eq. (26) will be denoted by $E$, so that

$$
E=\eta U(W-D)+(1-\eta) U(W)
$$

It follows that $t$ can be written as a function of $P$. This function will be denoted by $\varphi(P)$ :

$$
t=\varphi(P)=F^{-1}\left(\frac{U(W-P)-E}{U(W-P)-U(W-P-D)}\right) .
$$

The right hand side of (25) can also be written as a function of $P$ only, which leads to:

$$
\begin{aligned}
\hat{\Gamma}(P) & =P-(\eta-F(\varphi(P))) D \\
& =P-\left(\eta-\frac{U(W-P)-E}{U(W-P)-U(W-P-D)}\right) D .
\end{aligned}
$$

For full coverage, the optimal premium is equal to

$$
P=\varphi^{-1}(0)=W-U^{-1}(E)
$$


In view of this, the premium for a contract with any nonnegative probationary period can be written as

$$
P=\varphi^{-1}(t)=W-U^{-1}(E)-\epsilon D, \epsilon \geq 0
$$

Substituting into (29) gives, after some rewriting, the following result:

$$
\begin{aligned}
\hat{\Gamma}(P)= & \hat{\Gamma}\left(\varphi^{-1}(t)\right)=\hat{\Gamma}\left(W-U^{-1}(E)-\epsilon D\right) \\
= & W-U^{-1}(E)-\eta D \\
& +\left(\frac{(1-\epsilon) U\left(U^{-1}(E)+\epsilon D\right)+\epsilon U\left(U^{-1}(E)+(\epsilon-1) D\right)-E}{U(W-P)-U(W-P-D)}\right) D \\
\leq & W-U^{-1}(E)-\eta D+\left(\frac{U\left(U^{-1}(E)+\epsilon D+((1-\epsilon) 0-\epsilon) D\right)-E}{U(W-P)-U(W-P-D)}\right) D \\
= & W-U^{-1}(E)-\eta D=\hat{\Gamma}\left(W-U^{-1}(E)\right)=\hat{\Gamma}\left(\varphi^{-1}(0)\right) .
\end{aligned}
$$

The inequality sign follows from Jensen's inequality (which can be applied because $U(\cdot)$ is concave). It can only be replaced by an equality sign for $\epsilon=0$. Hence, offering full coverage is optimal.

\section{Acknowledgment}

The author is grateful to the referee for giving helpful comments, which have lead to a significant improvement of this paper. He also thanks Laura Ballotta.

\section{References}

EECKHOUDT, L., OUTREVILLE, J.F., LAUWERS, M., and CALCOEN, F. [1988]: "The Impact of a Probationary Period on the Demand of Insurance," The Journal of Risk and Insurance, 55, 219-228.

FLUET, C. [1992]: "Probationary Periods and Time-Dependent Deductibles in Insurance Markets with Adverse Selection," in Contributions to Insurance Economics, G. Dionne (Ed.), Kluwer Boston/Dordrecht/London, 359-375.

STIGLITZ, J. [1977]: “Monopoly, Non-Linear Pricing and Imperfect Information: The Insurance Market,” Review of Economic Studies, 44, 407-430. 